\title{
OPTIC NEURITIS DUE TO SOLVENT ABUSE
}

\author{
Carlos Alexandre Twardowschy', Hélio Afonso Ghizoni Teive ${ }^{2}$, Fábio Siquineli', \\ Arthur Furlaneto Fernandes', Ismael Paulo Búrigo', Arnolfo Carvalho-Neto ${ }^{3}$, Lineu César Werneck ${ }^{4}$
}

Solvent abuse is a public health problem in Brazil particularly among young adults and children. Inhalation of toluene-based products is popular with solvent sniffers because of the euphoric effect and easy availability of these substances. Chronic inhalation of toluene may result in a variety of neurologic complications like cerebellar dysfunction, optic atrophy, pyramidal tract signs, cranial nerve abnormalities. Also, personality changes, emotional instability and general cognitive decline have been attributed to its abuse?.

We report an unusual case of acute optic neuritis induced by thinner sniffing.

\section{CASE}

A 34-year-old man presented with an acute visual loss. He was admitted to the hospital reporting a sudden and progressive loss of visual acuity after sniffing a hole can of thinner during uninterrupted 48 hours. This homeless patient had a history of chronic solvent abuse for five years, moderate alcohol ingestion and tabagism, but denied other substances abuse. At the moment of the admission, 4 days after the beginning of the symptoms, he related to see only countenances. The physical examination and vital data were normal. The neurological examination was normal except for presenting bilateral mydriasis with severe reduction of the photomotor and consensual responses. Visual acuity was worse than 20/200. Ophthalmological examination demonstred bilateral papilledema, with normal ocular pressure and ocular movements. Other cranial nerves were normal. Laboratory examinations are listed in Table. Lumbar puncture showed $130 \mathrm{mmH}_{2} \mathrm{O}$ opening pressure, 27 red blood cells $/ \mathrm{mm}^{3}, 1$ leucocyte $/ \mathrm{mm}^{3}$, glucose concentration $75 \mathrm{mg} / \mathrm{dL}$, protein $39 \mathrm{mg} /$ $\mathrm{dL}$ and VDRL was negative. Magnetic resonance imaging (MRI) showed bilateral symmetric hyperintense lesions in the deep white matter, corpus callosum (splenium), centrum semiovale (Fig 1) and pons (Fig 2). EEG was irregular without specific pathological meanings. The patient was treated with methylprednisolone $1 \mathrm{gr} /$ day for three days associated with replacement of B complex vitamins. Six days after the admission, the patient ran away from the hospital and one month later returned in the neurology clinic relating important improvement of the visual acuity.

\section{DISCUSSION}

Today, organic solvents contained in industrial and domestic products are the most commonly abused volatile substances. Formerly, benzene was the major organic solvent in paints, lacquers, and thinners. However, benzene is toxic to bone marrow and liver and has been replaced by $\mathrm{n}$-hexane or toluene (methyl benzene), which are neuro-
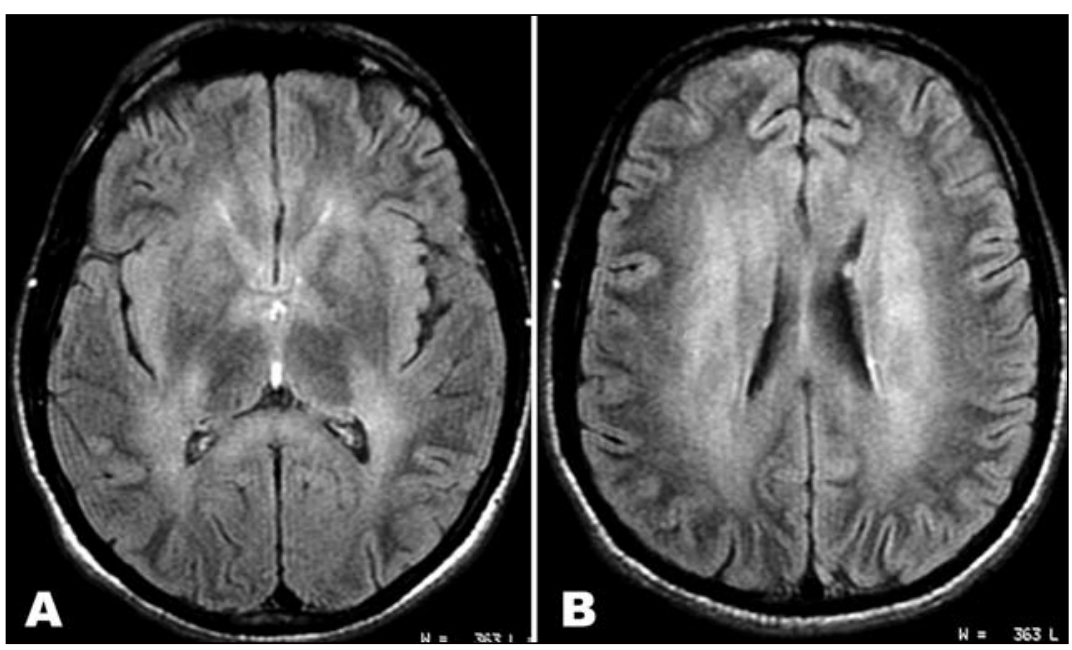

Fig 1. MRI. A) Axial FLAIR image shows bilateral symmetric hyperintense lesions in the deep white matter, corpus callosum (splenium) and B) centrum semiovale.

\section{NEURITE ÓPTICA RELACIONADA AO ABUSO DE SOLVENTE}

Neurology Service, Internal Medicine Department, Hospital de Clínicas, Federal University of Paraná, Curitiba PR, Brazil: 'Resident in Neurology; ${ }^{2}$ Adjunt Professor of Neurology; ${ }^{3}$ Adjunt Professor of Radiology; ${ }^{4}$ Full Professor of Neurology.

Received 1 August 2007, received in final form 1 November 2007. Accepted 4 December 2007.

Dr. Carlos Alexandre Twardowschy - Rua Dias da Rocha Filho 261 / 11 - 80040-050 Curitiba PR - Brasil. E-mail: carlos.a.tw@gmail.com 
Table. Patient's laboratory exams results.

\begin{tabular}{lccc}
\hline Exams & Results & Exams & Results \\
\hline Hemoglobin (g/dL) & 12.8 & HBsAG & Not reagent \\
MCV & 88 & Anti-HBC total & Not reagent \\
Leucocytes (per mm m $\left.^{3}\right)$ & 5000 & Anti-HBS & Not reagent \\
Platelets (per $\mathrm{mm}^{3}$ ) & 247000 & Anti-HCV & Not reagent \\
Creatinin (mg/dL) & 0.6 & VDRL & Not reagent \\
Urea (mg/dL) & 38 & HIV & Negative \\
Glucose (mg/dL) & 132 & PCR HSV & Negative \\
INR & 1.01 & PCR CMV & Negative \\
TSH (um/dL) & 2.09 & PCR VZV & Negative \\
ESR & 5 & PCR EBV & Negative \\
Total bilirubin (mg/dL) & 1.0 & Serum B12 & Normal \\
AST (mg/dL) & 22 & & \\
ALT (mg/dL) & 24 & & \\
Serum Ca (mg/dL) & 8.9 & & \\
Serum Na (mmol/L) & 143 & & \\
Serum K (mmol/L) & 3.5 & & \\
\hline MCV, mean corpuscular volume; TSH, thyroid stimulator hormone; ESR, erythrocyte sed- \\
imentation rate; INR, international normalized ratio; PCR HVS, polymerase chain reaction \\
for Herpes simplex virus; PCR VZ, polymerase chain reaction for Varicela-zoster virus; \\
PCR EBV, polymerase chain reaction for Epstein-Barr virus. &
\end{tabular}

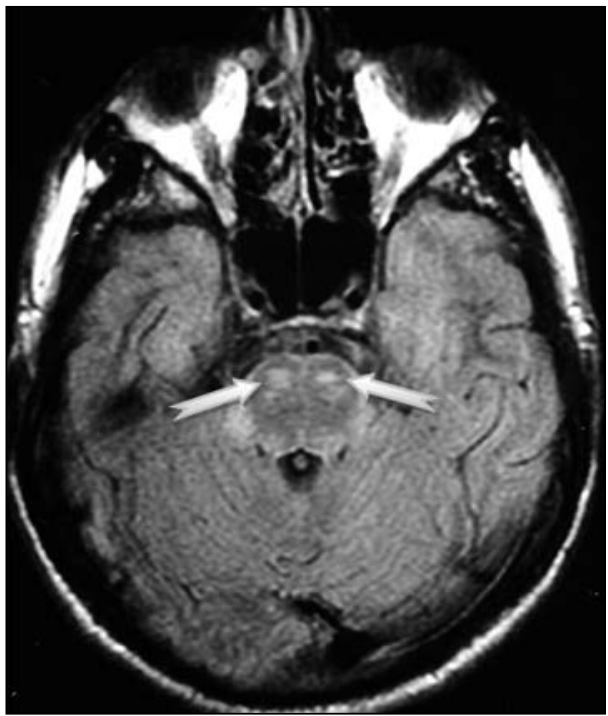

Fig 2. MRI. Axial FLAIR image shows bilateral symmetric hyperintense lesions in the base of the pons (arrows).

toxins. Toluene can cause multifocal neurologic disorders. Prior reports have been concerned with its acute effects'. Because of its lipophilicity, toluene rapidly penetrates into the central nervous system (CNS) after inhalation. Toxic toluene inhalation is most commonly the result of occupational exposure or recreational abuse.
A multiinstitutional study of 138 outpatients and inpatients with solvent dependence reported the organic solvents abused were "thinner" (a word often used as a general term for organic solvents for abuse), $69.6 \%$; pure toluene, $40.6 \%$; and glues, $39 \%$, with multiple replies ${ }^{2}$.

Gas chromatographic analysis of the volatile solvents revealed that the paint thinner consist almost entirely of toluene (methyl benzene), with traces of xylene (dimethylbenzene). The aerosol products contain 59 to $61 \%$ toluene, with traces of xylene and $10 \%$ methylene chloride (dichloromethane), a halogenated hydrocarbon. The remainder are propellants (butane-isobutane), which escape as gas when sprayed on the rag and are rarely inhaled. The solvent fractions that are inhaled consisted primarily of toluene and traces of methylene chloride'. Multiple components in the mixtures may enhance the net toxicity in a synergistic or additive fashion. Inhaled volatile hydrocarbons are rapidly absorbed from the lungs. Being highly lipophilic, they most easily enter and are retained within the lipid-rich nervous system.

Toxic and deficiency optic neuropathies are due to toxic chemicals and nutritional deficiency. The visual symptoms (dyschromatopsia and progressive reduction of visual acuity) usually occur bilaterally, simultaneously and painlessly. Total blindness is unusual with the exception of that caused by methanol. Neurologic abnormalities were 
seen in $65 \%$ of patients with a history of chronic solvent vapor abuse for 2 years or more. The abnormalities were cognitive $(60 \%)$, pyramidal $(50 \%)$, cerebellar $(45 \%)$, cranial nerve brainstem (25\%) and tremor (15\%).

We found five other reports of optic neuritis induced by thinner sniffing ${ }^{3-6}$. Like in our case, after they became conscious of their symptoms of slight visual disturbance, they continued to sniffing until they lost their vision. Another similar feature was lack of pain and response to steroids plus vitamin $B$ complex ${ }^{3}$. The MRI exam did not reveal any signs of optic nerve lesion (Figs 1A, 1B, 2).

The pathogenesis of the MRI lesions in toluene toxicity is poorly understood. Because of its high lipid solubility, toluene accumulates in lipid-rich tissues such as brain. Demyelination and gliosis in the cerebral and cerebellar white matter are the histologic changes reported in chronic toluene abusers ${ }^{7,8}$.

The cases with diffuse white matter change had a longer duration of abuse and obvious brain atrophy'. All patients with white matter changes had neurologic deficit. The association of white matter changes with abuse longer than 4 years suggests that white matter lesions are the result of a cumulative toxic effect of inhaled toluene ${ }^{8,11}$.

The main MRI findings in the CNS of the chronic solvent abusers are the white matter change on $\mathrm{T} 2$-weighted and proton density-weighted images as the patient reported presents (Figures). This finding is considered to represent the damage in myelin, such as demyelination or myelin pallor, reported in histopathologic reports 9 .

Periventricular white matter and the centrum semiovale were the most common locations for white matter changes in $19(46 \%)$ of 41 patients, thinning of the corpus callosum was revealed in nine patients (22\%), T2-weighted images revealed symmetric hypointensity in the thalami in eight patients (20\%). Some investigators have suggested that iron deposition and the partition of toluene into the lipids of cell membranes explain this finding ${ }^{10}$. T2-weighted images showed restricted white matter changes in 10 (53\%) of 19 patients with abnormal MRI imaging findings ". Computadorized tomography revealed difuse atrophy of cerebral hemispheres'.

So far, whether diffuse white matter change is caused by the spreading of restricted white matter change is not clear, neither is the reversibility of the white matter change.

Finally, in atypical visual disturbances, several laboratories, neurophysiological and neuroradiological tests are mandatory. Moreover, since pharmacological and nutritional therapies generally only provide a mild degree of symptomatic improvement, further studies are needed in optic neuritis due to solvent abuse.

\section{REFERENCES}

1. Hormes JT, Filley CM, Rosenberg NL. Neurologic sequelae of chronic solvent vapor abuse. Neurology 1986;36:698-702.

2. Fukui S, Wada K, Iyo M. Clinical characteristics of the recent organic solvent dependents who visited psychiatric facilities [in Japanese with English abstract]. J Men Health 1989;35:107-131.

3. Shinya H, Hoshino K, Kiritohshi M, Kiuchi S, Yamagami K, Nakatani T. [2 cases of acute retrobulbar neuritis by thinner inhalation; detected methanol of high concentration in gas phase assay] Chudoku Kenkyu. 2003;16:329-333.

4. Kohriyama K, Hori H, Murai Y, Ninomiya H, Tsukamoto Y. [Optic neuropathy induced by thinner sniffing] J UOEH 1989;11:449-453.

5. Ogawa Y, Takatsuki R, Uema T, et al. Acute optic neuropathy induced by thinner sniffing: inhalation of mixed organic solvent containing methyl alcohol and methyl acetate. Ind Health 1988;26:239-244.

6. Berg EF. Retrobulbar neuritis: a case report of presumed solvent toxicity. Ann Ophthalmol 1971;3:1351.

7. Rosenberg NL, Kleinschmidt BK, Davis KA, Dreisbach JN, Hormes JT, Filley CM. Toluene abuse causes diffuse central nervous system white matter changes. Ann Neurol 1988;23:611-614.

8. Damasceno BP, Capitani EM. Cerebellar atrophy related to chronic exposure to toluene: case report. Arq Neuropsiquiatr 1994:52:90-92.

9. Escobar A, Aruffo C. Chronic thinner intoxication: clinico-pathologic report of a human case. J Neurol Neurosurg Psychiatry 1980;43:986-994.

10. Yamanouchi N, Okada S, Kodama K, et al. White matter changes caused by chronic solvent abuse. Am J Neuroradiol 1995;16:1643-1649.

11. Aydin K, Sencer S, Demir T, Ogel K, Tunaci A, Minareci O. Cranial MR findings in chronic toluene abuse by inhalation. Am J Neuroradiol 2002;23:1173-1179. 
This article has received corrections in agreement with the ERRATUM published in Volume 66 Number 2a. 